\title{
Network Coherence in a Family of Book Graphs
}

\author{
Jing Chen ${ }^{1}$, Yifan $L i^{2}$ and Weigang Sun ${ }^{2 *}$ \\ ${ }^{1}$ School of Information Technology, Zhejiang Yuying College of Vocational Technology, Hangzhou, China, ${ }^{2}$ School of \\ Sciences, Hangzhou Dianzi University, Hangzhou, China
}

In this paper, we study network coherence characterizing the consensus behaviors with additive noise in a family of book graphs. It is shown that the network coherence is determined by the eigenvalues of the Laplacian matrix. Using the topological structures of book graphs, we obtain recursive relationships for the Laplacian matrix and Laplacian eigenvalues and further derive exact expressions of the network coherence. Finally, we illustrate the robustness of network coherence under the graph parameters and show that the parameters have distinct effects on the coherence.

Keywords: consensus, coherence, book graph, Laplacian spectra, recursive

\section{OPEN ACCESS}

Edited by:

Jia-Bao Liu,

Anhui Jianzhu University, China

Reviewed by:

Junhao Peng,

Guangzhou University, China

Zhongjun Ma,

Guilin University of Electronic

Technology, China

Yu Sun,

Jiangsu University, China

${ }^{*}$ Correspondence:

Weigang Sun

qdswg@163.com

Specialty section:

This article was submitted to Mathematical and Statistical Physics,

a section of the journal

Frontiers in Physics

Received: 15 July 2020

Accepted: 08 September 2020

Published: 16 October 2020

Citation:

Chen J, Li Y and Sun W (2020)

Network Coherence in a Family of

Book Graphs. Front. Phys. 8:583603.

doi: 10.3389/fphy.2020.583603

\section{INTRODUCTION}

With the discovery of deterministic small-world [1] and scale-free [2] networks, deterministically growing network models have gained increasing attention because they can provide exact results for topology and dynamics. As a special type of deterministic networks, fractal networks constructed by fractal structures, such as Koch fractals [3], Sierpinski fractals [4], and Vicsek fractals [5], have been widely studied. Presently the main issues that require consideration in fractal networks include random walks [6-9], consensus dynamics [10, 11] and percolation [12]. It is proved that fractal networks are good candidate network models for verifying the results of random graphs.

Calculating the Laplacian spectrum of a network plays an important role in the study of network characteristics. For example, the Kirchhoff index and global mean first-passage time of a network are related to the sum of reciprocals of non-zero eigenvalues [13-15]. The synchronizability [16] of a network refers to the ratio of the second smallest eigenvalue to the largest eigenvalue of the Laplacian matrix. In addition, the effective graph resistance is connected with the Laplacian spectrum [17]. Recently, network coherence [10] was introduced to characterize the extent of consensus of coupled agents under the noisy circumstance and was determined by the Laplacian spectrum in an $\mathrm{H}_{2}$ norm. This concept of the network coherence helps to study the relationship between the Laplacian eigenvalues and network consistency. Great progress has been made for some special networks such as Vicsek fractals [10], tree-like networks [11], Sierpiński graphs [18] and weighted networks [19]. Many works have been devoted to studying the network coherence. Hong et al. studied the role of Laplacian energy on the coherence in a family of tree-like networks with controlled initial states [20]. Patterson and Bamieh investigated the leader-follower coherence and proposed optimal algorithms to select the leaders [21]. Later, Sun et al. proposed a leader centrality to identify more influential spreaders using the optimal coherence [22].

It is known that the topology of a graph dominates the Laplacian eigenvalues [23]. Thus, calculating the Laplacian eigenvalues is a technical challenge and it is theoretical and practical interest to find new ways to calculate them. In this paper, a family of book graphs is chosen as our network models. The topological indices, e.g., randic index, sum connectivity index, geometricarithmetic index, fourth atom-bond connectivity index, and edge labeling, have been analytically 


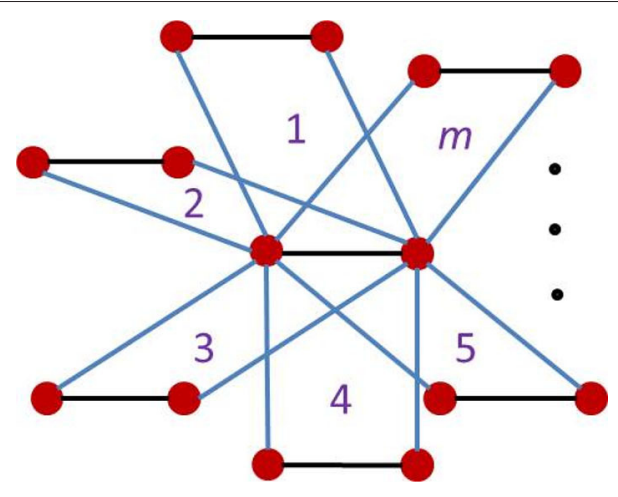

FIGURE 1 | Book graphs $B_{m}$.

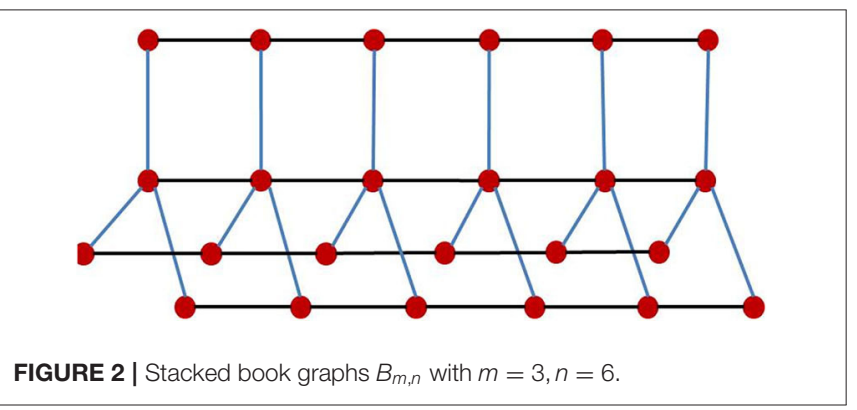

obtained $[24,25]$. However, the dynamics of the book graphs remains less understood, in spite of the facts that studying the dynamical processes leads to a better understanding of how the underlying systems work.

The rest of this paper is organized as follows. Book graphs and network coherence are presented in section 2. Section 3 gives detailed calculations of network coherence. Conclusions are given in section 4.

\section{MODEL PRESENTATION AND NETWORK COHERENCE}

\subsection{Book Graphs}

Book graphs $B_{m}$ are defined as the graph Cartesian product [26], i.e., $B_{m}=S_{m+1} \square P_{2}$, where $S_{m}(m \geq 1)$ is a star graph and $P_{2}$ is the path graph on two nodes, see Figure 1. The stacked book graphs $B_{m, n}$ of order $(m, n)$ are $B_{m, n}=S_{m+1} \square P_{n}$, where $P_{n}(n \geq 2)$ is the path graph on $n$ nodes, see Figure 2.

\subsection{Network Coherence}

The network coherence was introduced to characterize the steady-state variance of the deviation from consensus. The relationship [10] between network coherence and Laplacian eigenvalues was established. The consensus dynamics with the additive noise are given by

$$
\dot{x}_{i}(t)=-\sum_{j \in \Omega_{i}} L_{i j} x_{j}(t)+\eta_{i}(t)
$$

where $x_{i}(t)$ is the state of node $i$ and subject to the stochastic noise $\eta_{i}(t) . L$ is the Laplacian matrix. $\Omega_{i}$ is the neighboring node set of node $i$, and $\eta_{i}(t)$ is a delta-correlated Gaussian noise.

Then, the first-order network coherence is defined as the mean, steady-state variance of the deviation from the average of all node values, i.e.,

$$
H:=\frac{1}{N} \sum_{i=1}^{N} \lim _{t \rightarrow \infty} \operatorname{var}\left\{x_{i}(t)-\frac{1}{N} \sum_{j=1}^{N} x_{j}(t)\right\},
$$

where var is the expectation of the squared deviation of a random variable from its mean.

Let $0=\lambda_{1}<\lambda_{2} \leq \ldots \leq \lambda_{N}$ be the Laplacian eigenvalues. The network coherence is given by

$$
H=\frac{1}{2 N} \sum_{i=2}^{N} \frac{1}{\lambda_{i}}
$$

When the network has a smaller variance, it has a higher network coherence, meaning that it is more robust to the noise.

\section{CALCULATIONS OF NETWORK COHERENCE}

In this section, we present the detailed calculations of the sum of reciprocals of the Laplacian eigenvalues and obtain exact expressions of network coherence. According to the structure of $B_{m, n}$, its Laplacian matrix reads as

$$
L_{m, n}=\left(\begin{array}{cccccc}
L_{m}+I_{m+1} & -I_{m+1} & 0 & \cdots & 0 & 0 \\
-I_{m+1} & L_{m}+2 I_{m+1} & -I_{m+1} & \cdots & 0 & 0 \\
0 & -I_{m+1} & L_{m}+2 I_{m+1} & \cdots & 0 & 0 \\
\vdots & \vdots & \vdots & \vdots & \vdots & \vdots \\
0 & 0 & 0 & \cdots & L_{m}+2 I_{m+1} & -I_{m+1} \\
0 & 0 & 0 & \cdots & -I_{m+1} & L_{m}+I_{m+1}
\end{array}\right) \text {, }
$$

where $L_{m}$ is the Laplacian matrix of a star graph $S_{m}$, that is,

$$
L_{m}=\left(\begin{array}{cccc}
m & -1 & \cdots & -1 \\
-1 & 1 & \cdots & 0 \\
\vdots & \vdots & \vdots & \vdots \\
-1 & 0 & \cdots & 1
\end{array}\right)
$$

Then, we need to solve the characteristic equation $L_{m, n} x=\lambda x$, which is given by

$$
\begin{array}{cc}
\left(L_{m}+I_{m+1}\right) x_{1}-I_{m+1} x_{2} & =\lambda x_{1}, \\
-I_{m+1} x_{1}+\left(L_{m}+2 I_{m+1}\right) x_{2}-I_{m+1} x_{3} & =\lambda x_{2}, \\
\vdots & \vdots \\
-I_{m+1} x_{n-1}+\left(L_{m}+I_{m+1}\right) x_{n} & =\lambda x_{n}
\end{array}
$$

where $x=\left(x_{1}^{\mathrm{T}}, x_{2}^{\mathrm{T}}, \ldots, x_{n}^{\mathrm{T}}\right)^{\mathrm{T}}$ and the dimension of $x_{i}(1 \leq i \leq n)$ is $m+1$. 
Suppose $L_{m} x_{i}=\lambda_{j} x_{i}, i=1,2, \ldots, n$, where $\lambda_{j}(j=1,2, \ldots, m+1)$ are the eigenvalues of $L_{m}$. Then, Equation (2) becomes

$$
\begin{array}{cc}
\left(\lambda_{j}+1\right) x_{1}-x_{2} & =\lambda x_{1} \\
-x_{1}+\left(\lambda_{j}+2\right) x_{2}-x_{3} & =\lambda x_{2}, \\
\vdots \quad \vdots \quad \vdots & \\
-x_{n-1}+\left(\lambda_{j}+1\right) x_{n} & =\lambda x_{n} .
\end{array}
$$

We then rewrite Equation (3) as

$$
\left(R_{n}^{j}(\lambda)-\lambda_{j}\right) x_{1}=0, n \geq 2,
$$

where

$$
R_{n}^{j}(\lambda)=\lambda-1-\frac{1}{\lambda-\left(\lambda_{j}+2\right)-\frac{1}{\lambda-\left(\lambda_{j}+2\right)-\frac{1}{\cdots \lambda-\left(\lambda_{j}+2\right)-\frac{1}{\lambda-\left(\lambda_{j}+1\right)}}}} .
$$

Further, we have

$$
R_{n}^{j}(\lambda)=\lambda_{j}, j=1,2, \ldots, m+1
$$

We rewrite $R_{n}^{j}(\lambda)$ in a recursive form as

$$
\left\{\begin{array}{l}
R_{n}^{j}(\lambda)=\lambda-1-\frac{1}{R_{n-1}^{j}(\lambda)-\left(\lambda_{j}+1\right)}, \\
R_{2}^{j}(\lambda)=\lambda-1-\frac{1}{\lambda-\left(\lambda_{j}+1\right)}=\frac{\lambda^{2}-\left(2+\lambda_{j}\right) \lambda+\lambda_{j}}{\lambda-\left(\lambda_{j}+1\right)} .
\end{array}\right.
$$

From Equation (4), each eigenvalue $\lambda_{j}$ produces to $n$ eigenvalues and $B_{m, n}$ has $n(m+1)$ eigenvalues, denoted by $\Lambda_{n}=\left\{\lambda_{i}^{n} \mid 1 \leq i \leq\right.$ $n(m+1)\}=\Lambda_{n}^{1} \cup \Lambda_{n}^{2} \ldots \cup \Lambda_{n}^{m+1}$. For convenient calculations, we denote the smallest eigenvalues $\lambda_{1}^{n}=0$. In the following subsections, we divide $\lambda_{j}$ into two cases: $\lambda_{j} \neq 0$ and $\lambda_{j}=0$ to obtain the network coherence.

\subsection{When $\lambda_{j} \neq 0, j=2, \ldots, m+1$}

Let $R_{n}^{j}(\lambda)=T_{n}^{j}(\lambda) / P_{n}^{j}(\lambda)$, where $T_{n}^{j}(\lambda)$ and $P_{n}^{j}(\lambda)$ are two polynomials satisfying $\operatorname{gcd}\left[T_{n}^{j}(\lambda), P_{n}^{j}(\lambda)\right]=1$, the term $\operatorname{gcd}$ is the greatest common divisor. Then, we obtain the following recursive relationships as

$T_{n}^{j}(\lambda)=\left[T_{n-1}^{j}(\lambda)-\left(\lambda_{j}+1\right) P_{n-1}^{j}(\lambda)\right] \lambda-T_{n-1}^{j}(\lambda)+\lambda_{j} P_{n-1}^{j}(\lambda)$,

$P_{n}^{j}(\lambda)=T_{n-1}^{j}(\lambda)-\left(\lambda_{j}+1\right) P_{n-1}^{j}(\lambda)$,

where the initial conditions are

$$
\begin{aligned}
& T_{2}^{j}(\lambda)=\lambda^{2}-\left(2+\lambda_{j}\right) \lambda+\lambda_{j}, \\
& P_{2}^{j}(\lambda)=\lambda-\left(\lambda_{j}+1\right) .
\end{aligned}
$$

From Equation (5), we have

$$
\left\{\begin{array}{l}
t_{n}^{j}(0)=-t_{n-1}^{j}(0)+\lambda_{j} p_{n-1}^{j}(0), \\
p_{n}^{j}(0)=t_{n-1}^{j}(0)-\left(\lambda_{j}+1\right) p_{n-1}^{j}(0) .
\end{array}\right.
$$

where $t_{n}^{j}(0)$ and $p_{n}^{j}(0)$ are the constant terms of $T_{n}^{j}(\lambda)$ and $P_{n}^{j}(\lambda)$.

It follows from Equation (6) that

$$
p_{n}^{j}(0)+\left(\lambda_{j}+2\right) p_{n-1}^{j}(0)+p_{n-2}^{j}(0)=0 .
$$

Solving Equation (7) with initial conditions of $p_{2}^{j}(0)=-\left(\lambda_{j}+1\right)$ and $p_{3}^{j}(0)=\lambda_{j}^{2}+3 \lambda_{j}+1$ yields

$$
p_{n}^{j}(0)=c_{1}^{j}\left(r_{1}^{j}\right)^{g}+c_{2}^{j}\left(r_{2}^{j}\right)^{g},
$$

where $r_{1}^{j}$ and $r_{2}^{j}$ are the roots of the characteristic equation $\lambda^{2}+$ $\left(\lambda_{j}+2\right) \lambda+1=0$. The constants $r_{1}^{j}, r_{2}^{j}, c_{1}^{j}$ and $c_{2}^{j}$ are

$$
\left\{\begin{array}{l}
r_{1}^{j}=\frac{-\left(\lambda_{j}+2\right)+\sqrt{\lambda_{j}\left(\lambda_{j}+4\right)}}{2}, \\
r_{2}^{j}=\frac{-\left(\lambda_{j}+2\right)-\sqrt{\lambda_{j}\left(\lambda_{j}+4\right)}}{j_{j}^{2}}, \\
c_{1}=\frac{1}{\left(r_{1}^{j}\right)^{2}-1}\left[\left(\lambda_{j}\right)^{2}+3 \lambda_{j}+1+\left(\lambda_{j}+1\right) r_{2}^{j}\right], \\
c_{2}^{j}=\frac{1}{\left(r_{2}^{j}\right)^{2}-1}\left[\left(\lambda_{j}\right)^{2}+3 \lambda_{j}+1+\left(\lambda_{j}+1\right) r_{1}^{j}\right] .
\end{array}\right.
$$

Substituting Equation (8) into Equation (6) yields

$$
t_{n}^{j}(0)=-\left[c_{1}^{j}\left(r_{1}^{j}\right)^{n-2}\left(1+r_{1}^{j}\right)+c_{2}^{j}\left(r_{2}^{j}\right)^{n-2}\left(1+r_{2}^{j}\right)\right] .
$$

Next, we need to calculate the first-order terms $t_{n}^{j}(1), p_{n}^{j}(1)$ of $T_{n}^{j}(\lambda)$ and $P_{n}^{j}(\lambda)$. Using the relationship between $T_{n}^{j}(\lambda)$ and $P_{n}^{j}(\lambda)$ of Equation (5) gives

$$
\begin{aligned}
& t_{n}^{j}(1)=t_{n-1}^{j}(0)-\left(\lambda_{j}+1\right) p_{n-1}^{j}(0)-t_{n-1}^{j}(1)+\lambda_{j} p_{n-1}^{j}(1), \\
& p_{n}^{j}(1)=t_{n-1}^{j}(1)-\left(\lambda_{j}+1\right) p_{n-1}^{j}(1),
\end{aligned}
$$

where the initial values are $t_{2}^{j}(1)=-\left(\lambda_{j}+2\right), p_{2}^{j}(1)=1, p_{3}^{j}(1)=$ $-\left(2 \lambda_{j}+3\right)$. Then, we obtain

$$
\begin{aligned}
t_{n}^{j}(1) & =\left\{e_{j}\left(r_{1}^{j}\right)^{2}+\left[n g_{j}+\left(\lambda_{j}+1\right) e_{j}\right] r_{1}^{j}\right. \\
& \left.+(n-1)\left(\lambda_{j}+1\right) g_{j}\right\}\left(r_{1}^{j}\right)^{n-2} \\
& +\left\{f_{j}\left(r_{2}^{j}\right)^{2}+\left[n h_{j}+\left(\lambda_{j}+1\right) f_{j}\right] r_{2}^{j}\right. \\
& \left.+(n-1)\left(\lambda_{j}+1\right) h_{j}\right\}\left(r_{2}^{j}\right)^{n-2}, \\
p_{n}^{j}(1) & =e_{j}\left(r_{1}^{j}\right)^{n-1}+f_{j}\left(r_{2}^{j}\right)^{n-1}+(n-1)\left[g_{j}\left(r_{1}^{j}\right)^{n-2}+h_{j}\left(r_{2}^{j}\right)^{n-2}\right],
\end{aligned}
$$


where

$$
\left\{\begin{array}{l}
g_{j}=-\frac{c_{1}^{j}\left[\left(\lambda_{j}+2\right) r_{1}^{j}+1\right]}{2\left(r_{1}^{j}\right)^{2}+\left(\lambda_{j}+2\right) r_{1}^{j}}, \\
h_{j}=-\frac{c_{2}^{j}\left[\left(\lambda_{j}+2\right) r_{2}^{j}+1\right]}{2\left(r_{2}^{j}\right)^{2}+\left(\lambda_{j}+2\right) r_{2}^{j}}, \\
e_{j}=\frac{\left[1-\left(g_{j}+h_{j}\right)\right] r_{2}^{j}+2\left(g_{j} r_{1}^{j}+h_{j} r_{2}^{j}\right)+\left(2 \lambda_{j}+3\right)}{1-\left(r_{1}^{j}\right)^{2}}, \\
f_{j}=\frac{\left[1-\left(g_{j}+h_{j}\right)\right] r_{1}^{j}+2\left(g_{j} r_{1}^{j}+h_{j} r_{2}^{j}\right)+\left(2 \lambda_{j}+3\right)}{1-\left(r_{2}^{j}\right)^{2}} .
\end{array}\right.
$$

We introduce a new polynomial as

$$
\begin{aligned}
D_{n}^{j}(\lambda) & =T_{n}^{j}(\lambda)-\lambda_{j} P_{n}^{j}(\lambda) \\
& =\left(\lambda-\lambda_{(j-1) n+1}^{n}\right)\left(\lambda-\lambda_{(j-1) n+2}^{n}\right) \ldots\left(\lambda-\lambda_{j n}^{n}\right) .
\end{aligned}
$$

Using the Vieta's formula $[26,27]$ for $D_{n}^{j}(\lambda)=0$, we obtain its constant and first-order terms, denoted by $d_{n}^{j}(0), d_{n}^{j}(1)$, that is,

$$
\left\{\begin{aligned}
d_{n}^{j}(0)= & t_{n}^{j}(0)-\lambda_{j} p_{n}^{j}(0) \\
= & -c_{1}^{j}\left(r_{1}^{j}\right)^{n-2}\left[1+\left(1+\lambda_{j}\right) r_{1}^{j}\right] \\
- & c_{2}^{j}\left(r_{2}^{j}\right)^{n-2}\left[1+\left(1+\lambda_{j}\right) r_{2}^{j}\right], \\
d_{n}^{j}(1)= & t_{n}^{j}(1)-\lambda_{j} p_{g}^{j}(1) \\
= & \left(r_{1}^{j}\right)^{n-2}\left[e_{j}\left(r_{1}^{j}\right)^{2}+\left(n g_{j}+e_{j}\right) r_{1}^{j}+(n-1) g_{j}\right] \\
& +\left(r_{2}^{j}\right)^{n-2}\left[f_{j}\left(r_{2}^{j}\right)^{2}+\left(n h_{j}+f_{j}\right) r_{2}^{j}+(n-1) h_{j}\right] .
\end{aligned}\right.
$$

\subsection{When $\lambda_{j}=0$}

When $\lambda_{j}=0, R_{n}^{1}(\lambda)=0$ has only one root $\lambda_{1}^{n}=0$. To obtain all the non-zero roots of $R_{n}^{j}(\lambda)=0$, we introduce a new polynomial, i.e.,

$$
Z_{n}^{1}(\lambda)=\frac{1}{\lambda} R_{n}^{1}(\lambda)
$$

Further,

$$
\begin{aligned}
& T_{n}^{1}(\lambda)=(\lambda-1) T_{n-1}^{1}(\lambda)-P_{n-1}^{1}(\lambda), \\
& P_{n}^{1}(\lambda)=\lambda T_{n-1}^{1}(\lambda)-P_{n-1}^{1}(\lambda),
\end{aligned}
$$

where the initial conditions are $T_{2}^{1}(\lambda)=\lambda-2, P_{2}^{1}(\lambda)=\lambda-1$. In the same way, we obtain the following coefficients, which are given by

$$
\begin{aligned}
& \left\{\begin{array}{l}
t_{n}^{1}(0)=(-1)^{n-1} n, \\
t_{n}^{1}(1)=(-1)^{n-2} \cdot \frac{n\left(n^{2}-1\right)}{6},
\end{array}\right. \\
& \left\{\begin{array}{l}
p_{n}^{1}(0)=(-1)^{n-2}, \\
p_{n}^{1}(1)=(-1)^{n-2} \cdot \frac{n(n-1)}{2},
\end{array}\right.
\end{aligned}
$$

It follows from Equation (9) that

$$
\left\{\begin{aligned}
d_{n}^{1}(0) & =(-1)^{n-1} \lambda_{2}^{n} \lambda_{3}^{n} \ldots \lambda_{n}^{n} \\
& =(-1)^{n-1} n \\
d_{n}^{1}(1) & =(-1)^{n-2}\left[\lambda_{3}^{n} \lambda_{4}^{n} \ldots \lambda_{n}^{n}+\lambda_{2}^{n} \lambda_{4}^{n} \ldots \lambda_{n}^{n}+\ldots\right. \\
& \left.+\lambda_{2}^{n} \lambda_{3}^{n} \ldots \lambda_{n-1}^{n}\right] \\
& =(-1)^{n-2} \cdot \frac{n\left(n^{2}-1\right)}{6} .
\end{aligned}\right.
$$

\subsection{Exact Solution of Network Coherence for $B_{m, n}$}

We introduce a polynomial $D_{n}(\lambda)$ to obtain the exact solution of the network coherence, i.e.,

$$
D_{n}(\lambda)=\prod_{j=1}^{m+1} D_{n}^{j}(\lambda)=\prod_{i=2}^{n(m+1)}\left(\lambda-\lambda_{i}^{n}\right) .
$$

According to Equations (10) and (11), the constant and firstorder terms of $D_{n}(\lambda)$ are

$$
\begin{aligned}
d_{n}(0)= & \prod_{j=1}^{m+1} d_{n}^{j}(0), \\
d_{n}(1)= & \underbrace{d_{n}^{1}(1) d_{n}^{2}(0) \ldots d_{n}^{m+1}(0)}_{m+1} \\
& +\underbrace{d_{n}^{1}(0) d_{n}^{2}(1) \ldots d_{n}^{m+1}(0)}_{m+1}+\ldots+\underbrace{d_{n}^{1}(0) d_{n}^{2}(0) \ldots d_{n}^{m+1}(1)}_{m+1} .
\end{aligned}
$$

Based on the Vieta's theorem [26, 27], the network coherence reads as

$$
H=\frac{1}{2 N} \sum_{i=2}^{N} \frac{1}{\lambda_{i}}=-\frac{1}{2 N} \frac{d_{n}(1)}{d_{n}(0)} .
$$

When $m=3$, the Laplacian matrix $L_{m}$ has four eigenvalues, that is, $\lambda_{1}=0, \lambda_{2}=\lambda_{3}=1, \lambda_{4}=4$. Using the abovementioned calculations, we obtain the analytical expression of network coherence, i.e.,

$$
\begin{aligned}
H_{(n)}= & \frac{1}{8 n}\left\{\frac{n^{2}-1}{6}\right. \\
& -20 \frac{\left[\alpha_{1}+g_{2}\left(n r_{1}^{2}+n-1\right)\right]\left(r_{1}^{2}\right)^{n-2}+\left[\alpha_{2}+h_{2}\left(n r_{2}^{2}+n-1\right)\right]\left(r_{2}^{2}\right)^{n-2}}{\beta_{1}\left(r_{1}^{2}\right)^{n-2}+\beta_{2}\left(r_{2}^{2}\right)^{n-2}} \\
& \left.-2 \frac{\left[\theta_{1}+g_{4}\left(n r_{1}^{4}+n-1\right)\right]\left(r_{1}^{4}\right)^{n-2}+\left[\theta_{2}+h_{4}\left(n r_{2}^{4}+n-1\right)\right]\left(r_{2}^{4}\right)^{n-2}}{\eta_{1}\left(r_{1}^{4}\right)^{n-2}+\eta_{2}\left(r_{2}^{4}\right)^{n-2}}\right\},
\end{aligned}
$$

where $\alpha_{1}=-\frac{5-2 \sqrt{5}}{25}, \alpha_{2}=-\frac{5+2 \sqrt{5}}{25}, \beta_{1}=15-7 \sqrt{5}, \beta_{2}=$ $15+7 \sqrt{5}, \theta_{1}=-\frac{10-7 \sqrt{2}}{32}, \theta_{2}=-\frac{10+7 \sqrt{2}}{32}, \eta_{1}=24-17 \sqrt{2}$, $\eta_{2}=24+17 \sqrt{2}, g_{2}=-\frac{\left.(5-\sqrt{5})\left(3 r_{1}^{2}+1\right)\right)}{10 r_{1}^{2}\left(2 r_{1}^{2}+3\right)}, h_{2}=-\frac{\left.(5+\sqrt{5})\left(3 r_{2}^{2}+1\right)\right)}{10 r_{2}^{2}\left(2 r_{2}^{2}+3\right)}$, $g_{4}=-\frac{\left.(2-\sqrt{2})\left(6 r_{1}^{4}+1\right)\right)}{8 r_{1}^{4}\left(r_{1}^{4}+3\right)}, h_{4}=-\frac{\left.(2+\sqrt{2})\left(6 r_{2}^{4}+1\right)\right)}{8 r_{2}^{4}\left(r_{2}^{4}+3\right)}, r_{1}^{2}=\frac{-3+\sqrt{5}}{2}$, $r_{2}^{2}=\frac{-3-\sqrt{5}}{2}, r_{1}^{4}=-3+2 \sqrt{2}, r_{2}^{4}=-3-2 \sqrt{2}$.

\subsection{Exact Solution of Network Coherence for $B_{m}$}

To investigate the effect of the parameters $m$ on the network coherence, we propose another method to obtain the solution regarding the parameters $m$. When $n=2$, the Laplacian matrix is

$$
L_{m, 2}=\left(\begin{array}{cc}
L_{m} & -I_{m+1} \\
-I_{m+1} & L_{m}
\end{array}\right) \text {. }
$$



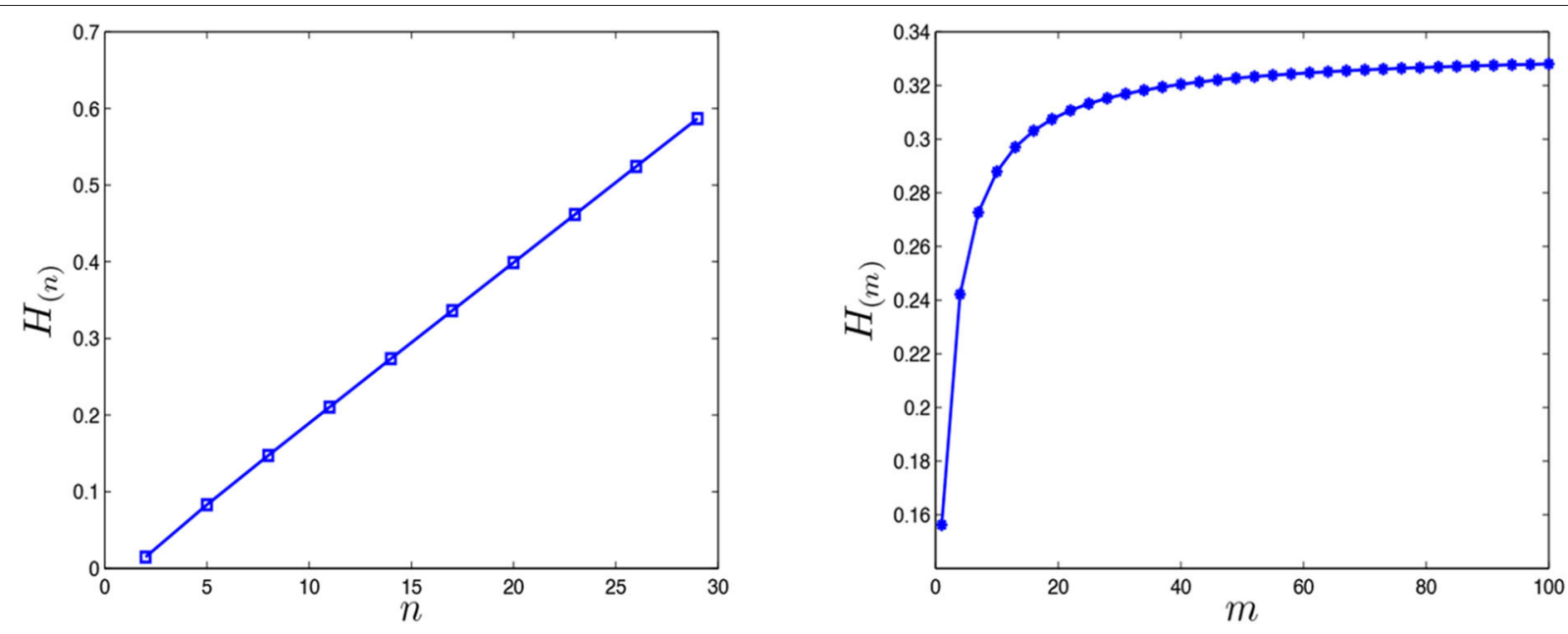

FIGURE 3 | Network coherence regarding the parameters $n$ and $m$.

Then, the characteristic polynomial $P(\lambda)$ of $L_{m, 2}$ is

$$
\begin{aligned}
P(\lambda) & =\left|\begin{array}{cc}
L_{m}-\lambda I_{m+1} & -I_{m+1} \\
-I_{m+1} & L_{m}-\lambda I_{m+1}
\end{array}\right| \\
& =\left|L_{m}-(\lambda+1) I\right| \cdot\left|L_{m}-(\lambda-1) I\right| \\
& =\lambda(\lambda-2)(\lambda-m-1)(\lambda-m-3)(\lambda-1)^{m-1}(\lambda-3)^{m-1} .
\end{aligned}
$$

The roots of this polynomial $P(\lambda)$ are as follows,

$$
\{0,2, m+1, m+3, \underbrace{1, \ldots, 1}_{m-1}, \underbrace{3, \ldots, 3}_{m-1}\} \text {. }
$$

By the definition (1), we finally obtain the network coherence with regard to the parameters $m$, which is given by

$$
H_{(m)}=\frac{1}{4 m+4}\left[\frac{1}{2}+\frac{1}{m+1}+\frac{1}{m+3}+\frac{4(m-1)}{3}\right] .
$$

From the expressions (12) and (13), we plot the relationships between network coherence and the parameters $m$ and $n$, see Figure 3. It shows that the values of network coherence linearly increase with $n$, while the network coherence will achieve a steady constant state for a large $m$, i.e., $H_{(m)} \rightarrow \frac{1}{3}$, meaning that the consensus displays worse with increasing values of $n$. In a word, the number of nodes $n$ in the path graph has more influence than the number of nodes $m$ in the star graph.

\section{CONCLUSIONS}

In this paper, we have studied the consensus problems in noisy book graphs. Using the graph's constructions, we have

\section{REFERENCES}

1. Comellas F, Ozon J, Peters JG. Deterministic small-world communication networks. Inf Process Lett. (2000) 76:83-90. doi: 10.1016/S0020-0190(00)00118-6 obtained the recursive relationships for the Laplacian matrix and Laplacian eigenvalues and proposed a method to derive exact expressions of the sum of reciprocals of these eigenvalues. We then have presented exact solutions of network coherence with regard to graph parameters and investigated their effects on the coherence. It is shown that the larger size of star graphs results in better consensus, while the larger size of path graphs leads to worse consensus. The obtained results showed that the structure difference produces distinct performance on the coherence. Our method for the book graphs could be applied to study their random walks and Kirchhoff index.

\section{DATA AVAILABILITY STATEMENT}

The raw data supporting the conclusions of this article will be made available by the authors, without undue reservation.

\section{AUTHOR CONTRIBUTIONS}

JC, YL, and WS contributed to the conception and design of the study. JC and YL performed the analytical and numerical results. JC and WS wrote the manuscript. All authors contributed to the manuscript revision, read, and approved the submitted version. All authors contributed to the article and approved the submitted version.

\section{FUNDING}

This work was supported by the Zhejiang Provincial Natural Science Foundation of China (No. LY20F030007).

2. Barabási AL, Ravasz E, Vicsek T. Deterministic scale-free networks. Phys A. (2001) 299:559-64. doi: 10.1016/S0378-4371(01)00369-7

3. Dai MF, Li XY, Xi LF. Random walks on non-homogenous weighted Koch networks. Chaos. (2013) 23:033106. doi: 10.1063/1. 4810927 
4. Wang SJ, Yu ZY, Xi LF. Average geodesic distance of Sierpinski gasket and Sierpinski networks. Fractals. (2017) 25:1750044. doi: 10.1142/S0218348X1750044X

5. Zhang ZZ, Wu B, Zhang HJ, Zhou SG, Guan JH, Wang ZG. Determining global mean-first-passage time of random walks on Vicsek fractals using eigenvalues of Laplacian matrices. Phys Rev E. (2010) 81:031118. doi: 10.1103/PhysRevE.81.031118

6. Condamin S, Bénichou O, Tejedor V, Voituriez R, Klafter J. Firstpassage times in complex scale-invariant media. Nature. (2007) 450: 77-80. doi: 10.1038 /nature06201

7. Wu ZK, Hou BY, Zhang HJ, Jin F. Scaling of average weighted shortest path and average receiving time on weighted expanded Koch networks. Int J Mod Phys B. (2014) 28:1450111. doi: 10.1142/S0217979214501112

8. Peng JH, Agliari E, Zhang, ZZ. Exact calculations of first-passage properties on the pseudofractal scale-free web. Chaos. (2015) 25:073118. doi: $10.1063 / 1.4927085$

9. Peng JH, Agliari E. Exact results for the first-passage properties in a class of fractal networks. Chaos. (2019) 29:023105. doi: 10.1063/1.5080481

10. Patterson S, Bamieh B. Consensus and coherence in fractal networks. IEEE Trans Control Netw Syst. (2014) 1:338-48. doi: 10.1109/TCNS.2014.2357552

11. Sun WG, Ding QY, Zhang JY, Chen FY. Coherence in a family of tree networks with an application of Laplacian spectrum. Chaos. (2014) 24:043112. doi: $10.1063 / 1.4897568$

12. Balankin AS, Martinez-Cruz MA, Susarrey-Huerta O, Adame LD. Percolation on infinitely ramified fractal networks. Phys Lett A. (2018) 382:12-9. doi: $10.1016 /$ j.physleta.2017.10.035

13. Klein DJ and Randić M. Resistance distance. J Math Chem. (1993) 13:81-95. doi: $10.1007 / \mathrm{BF} 01164627$

14. Bonchev D, Balaban AT, Liu X, Klein DJ. Molecular cyclicity and centricity of polycyclic graphs. I. Cyclicity based on resistance distances or reciprocal distances. Int J Quant Chem. (1994) 50:1-20. doi: 10.1002/qua.560500102

15. Wang XQ, Dai MF, Chen YF, Zong Y, Sun Y, Su, WY. Determining entire mean first-passage time for Cayley networks. Int J Mod Phys C. (2018) 29:1850009. doi: 10.1142/S0129183118500092

16. Duan ZS, Chen GR, Huang L. Complex network synchronizability: analysis and control. Phys Rev E. (2007) 76:056103. doi: 10.1103/PhysRevE.76.056103

17. Ellens W, Spieksma FM, Van Mieghem P, Jamakovic A, Kooij RE. Effective graph resistance. Linear Algebra Appl. (2011) 435:2491-506. doi: 10.1016/j.laa.2011.02.024
18. Qi Y, Zhang ZZ, Yi Y, Li H. Consensus in self-similar hierarchical graphs and Sierpiński graphs: Convergence speed, delay robustness, and coherence. IEEE Trans Cybern. (2019) 49:592-603. doi: 10.1109/TCYB.2017.2781714

19. Dai MF, He JJ, Zong Y, Ju TT, Sun Y, Su WY. Coherence analysis of a class of weighted networks. Chaos. (2018) 28:043110. doi: 10.1063/1.4997059

20. Hong MD, Sun WG, Liu SY, Xuan TF. Coherence analysis and Laplacian energy of recursive trees with controlled initial states. Front Inform Technol Elect Eng. (2020) 21:931-8. doi: 10.1631/FITEE.1900133

21. Patterson S, Bamieh B. Leader selection for optimal network coherence. In: Proceedings of the 49th IEEE Conference on Decision and Control. Atlanta, GA (2010). p. 2692-7. doi: 10.1109/CDC.2010.5718151

22. Sun WG, Sun MT, Guan JB, Jia Q. Robustness of coherence in noisy scale-free network and applications to identification of influential spreaders. IEEE Trans Circuits Syst II. (2020) 67:1274-8. doi: 10.1109/TCSII.2019.29 29139

23. Grone R, Merris R, Sunder VS. The Laplacian spectrum of a graph. SIAM J Matrix Anal Appl. (1990) 11:218-38. doi: 10.1137/06 11016

24. Khalid R, Idrees N, Saif MJ. Topological characterization of book graph and stacked book graph. Comput Mater Con. (2019) 60:41-54. doi: $10.32604 / \mathrm{cmc} .2019 .06554$

25. Daoud SN, Elsawy AN. Edge even graceful labelling of some book graphs. $J$ Taibah Univ Sci. (2018) 12: 315-30. doi: 10.1080/16583655.2018.1469292

26. Beineke LW, Wilson RJ, Cameron PJ. Topics in Algebraic Graph Theory. New York, NY: Cambridge University Press (2004).

27. Comellas F, Miralles A. Mean first-passage time for random walks on generalized deterministic recursive trees. Phys Rev E. (2010) 81:061103. doi: $10.1103 /$ PhysRevE.81.061103

Conflict of Interest: The authors declare that the research was conducted in the absence of any commercial or financial relationships that could be construed as a potential conflict of interest.

Copyright (๑) 2020 Chen, Li and Sun. This is an open-access article distributed under the terms of the Creative Commons Attribution License (CC BY). The use, distribution or reproduction in other forums is permitted, provided the original author(s) and the copyright owner(s) are credited and that the original publication in this journal is cited, in accordance with accepted academic practice. No use, distribution or reproduction is permitted which does not comply with these terms. 